\title{
Respuesta dietaria de tres rapaces frente a una presa introducida en Patagonia
}

\author{
Dietary response of three raptor species to an introduced prey in Patagonia
}

ANA L. MONSERRAT ${ }^{1 *}$, MARTÍN C. FUNES ${ }^{2} \&$ ANDRÉS J. NOVARO 3

\author{
${ }^{1}$ Laboratorio de Geología Marina y Dinámica de Costas, Departamento de Geología, Facultad de Ciencias Exactas y \\ Naturales, Universidad de Buenos Aires, Pabellón II, Ciudad Universitaria, C1428EHA, Buenos Aires, Argentina \\ ${ }^{2}$ Departamento de Fauna Terrestre, Centro de Ecología Aplicada del Neuquén, \\ Casilla de Correos 7, Junín de los Andes, 8371, Argentina \\ ${ }^{3}$ CONICET - Wildlife Conservation Society, Centro de Ecología Aplicada del Neuquén, \\ Casilla de Correos 7, Junín de los Andes, 8371, Argentina \\ *Corresponding author: e-mail: analaura@gl.fcen.uba.ar
}

\begin{abstract}
RESUMEN
Los depredadores de marcada respuesta funcional pueden poseer la capacidad de estabilizar las poblaciones de sus presas, lo cual es destacable en el caso de presas introducidas, que suelen causar impactos negativos en los ecosistemas nativos y las actividades agropecuarias. La situación actual de la Patagonia es crítica respecto a la introducción de especies. Estudios previos indican que los depredadores nativos exhiben un cambio general en sus dietas debido a la introducción de presas exóticas que están reemplazando a sus presas originales. Determinamos los hábitos alimenticios del águila (Geranoaetus melanoluecus), el búho magallánico (Bubo magellanicus) y el aguilucho común (Buteo polyosoma), tres de las principales aves depredadoras de la liebre europea (Lepus europaeus), introducida en el noroeste de Patagonia. Para esto analizamos 321, 115 y 78 egagrópilas de águila, búho y aguilucho respectivamente. Evaluamos las respuestas funcionales a la densidad de liebre europea y comparamos las dietas entre sitios diferentes. Las principales presas del aguilucho y del búho fueron los roedores sigmodontinos y en segundo lugar los tuco-tucos (Ctenomys spp.). El águila consumió principalmente liebres y en segundo lugar tuco-tucos, presentando una respuesta funcional significativa para la presa introducida. La dieta de este rapaz fue diferente entre sitios con alta y baja densidad de liebres. Para los otros dos rapaces no encontramos una respuesta funcional significativa para la liebre europea, pero la dieta del aguilucho varió en función del sitio geográfico. Concluimos que tanto el águila mora como el aguilucho se alimentan de manera generalista, siendo el búho el único de los tres rapaces con tendencia especialista sobre pequeños roedores. Por otra parte, la variación de la dieta del águila en función de la densidad de liebre europea le brinda el potencial de contribuir a la regulación de las poblaciones de la misma.
\end{abstract}

Palabras clave: respuesta funcional, rapaces, águila mora, búho, aguilucho, liebre europea, Patagonia.

\begin{abstract}
Predators with strong functional responses may be able to help stabilize prey population levels. This is worth noting mainly in the case of introduced prey, whose impact on native ecosystems often involves significant economic and environmental damage. The current Patagonian situation is critical with respect to introduced species. Previous studies have shown that native vertebrate predators are able to change their feeding patterns, switching from native to exotic prey. We assessed the food habits of the grey buzzard-eagle (Geranoaetus melanoleucus), the Magellanic horned owl (Bubo magellanicus), and the red-backed hawk (Buteo polyosoma), the three most important avian predators of the introduced European hare (Lepus europaeus) in north-western Patagonia. We analysed 321, 115 and 78 eagle, owl, and hawk pellets respectively. We evaluated their functional responses to hare densities and compared their diets between different sites. Sigmodontine rodents were the hawk's and owl's main prey, followed by the tuco-tuco (Ctenomys spp.). The eagle consumed mainly European hares and then tuco-tucos, showing a significant functional response to the introduced prey. This raptor also had different diets in sites with high and low hare density. The other two raptors did not show a significant functional response to hare densities. However, the hawk's diet varied according to geographic location. We conclude that eagles and hawks are generalist predators, whereas the owl tends to specialize on small mammals. The changes in the eagle's diet in relation to European hare densities provide the potential to contribute to regulate the hare population.
\end{abstract}

Keywords: functional response, raptors, grey buzzard-eagle, red -backed hawk, Magellanic owl, European hare, Patagonia. 


\section{INTRODUCCIÓN}

Los depredadores pueden responder a cambios en la disponibilidad de sus presas a través de variaciones en sus dietas (respuesta funcional) o en sus números poblacionales (respuesta numérica). La respuesta funcional de un depredador mide su tasa de consumo en función de la disponibilidad de la presa. Teóricamente toda curva de respuesta funcional alcanza un nivel de saturación a altas densidades de presas. Esto ocurre cuando el depredador se sacia, pero esta respuesta puede presentar diferentes formas, reconociéndose tres tipos principales de respuesta funcional: lineal, convexa y sigmoidea (Holling 1959). Una condición necesaria, pero no suficiente, para que un depredador pueda regular una población de presas es que la pendiente de la curva de respuesta funcional sea positiva (Solomon 1949). Las evidencias empíricas sugieren que los depredadores con marcada respuesta funcional, especialmente aquellos que pueden consumir presas alternativas con facilidad y por lo tanto suelen presentar respuesta sigmoidea, poseen mayor capacidad de estabilizar las poblaciones de sus presas (Erlinge et al. 1984, Schmitz 1995, Norrdahl \& Korpimäki 2000).

Los antecedentes en aves rapaces con respuestas funcionales son numerosos (Lindén \& Wikman 1983, Korpimäki \& Norrdahl 1989, 1991, Salamolard et al. 2000), pero no se ha encontrado un patrón general de respuesta. Poco se conoce, por otro lado, sobre interacciones entre depredadores y presas vertebrados cuando uno o ambos han sido introducidos y por lo tanto no han tenido una historia evolutiva común. El conocimiento de la dinámica de las interacciones predador-presa adquiere relevancia económica y de conservación cuando uno de ellos es exótico, pues suelen perjudicar las actividades productivas humanas y el estado de los ecosistemas. Tal es el caso, por ejemplo, de roedores y lagomorfos que han invadido ecosistemas productivos en todo el mundo (Davis et al. 2003, Wood \& Chung Gait Fee 2003).

La situación actual de la Patagonia es crítica respecto a la introducción de especies. Se ha encontrado, por ejemplo, que los depredadores nativos exhiben un cambio general en sus dietas debido a la introducción de presas exóticas que están reemplazando a sus presas originales (Franklin et al. 1999, Novaro et al. 2000). Particularmente, la liebre europea (Lepus europaeus), que fue introducida a fines del siglo diecinueve con propósitos de caza (Grigera \& Rapoport 1983), presenta elevadas densidades, especialmente en los ambientes más productivos de la Patagonia. La liebre europea tiene un importante impacto negativo sobre los hábitats y las actividades agropecuarias, por lo que es relevante investigar la posibilidad de su regulación por depredadores naturales.

En este trabajo analizamos la respuesta dietaria de tres rapaces nativas, el águila (Geranoaetus melanoleucus), el búho magallánico (Bubo magellanicus) y el aguilucho (Buteo polyosoma), ante la liebre europea introducida en el noroeste de la Patagonia. Estas rapaces son las tres principales aves depredadoras que se presumen capaces de cazar liebres europeas en la región (Jiménez \& Jaksic 1990, Jiménez \& Jaksic 1991, Pavez et al. 1992, Hiraldo et al. 1995, Donázar et al. 1997). A través de este estudio pretendemos profundizar el conocimiento sobre el comportamiento alimenticio de estos tres rapaces, analizando sus dietas en diferentes sitios y evaluando si presentan respuestas funcionales en el consumo de una presa introducida.

\section{MATERIALES Y MÉTODOS}

\section{Área de estudio}

El área de estudio se localiza en el noroeste de la Patagonia, provincia de Neuquén, Argentina

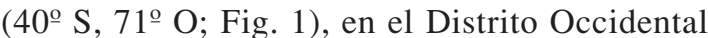
de la Provincia Fitogeográfica Patagónica (Cabrera 1976). El clima es seco y frío, con heladas durante todo el año. La temperatura media anual es de $11{ }^{\circ} \mathrm{C}$ y la precipitación media anual es de 280 a $1.000 \mathrm{~mm}$, se concentra durante el invierno y se incrementa de este a oeste.

El área de estudio abarca una superficie de $3.000 \mathrm{~km}^{2}$, con 12 sitios de muestreo en el sur de Neuquén y un sitio para el búho $330 \mathrm{~km}$ al norte (Fig. 1). Los sitios del sur de Neuquén que se encuentran al este en general son los más áridos e incluyen a Calcatre, Mesa, La 

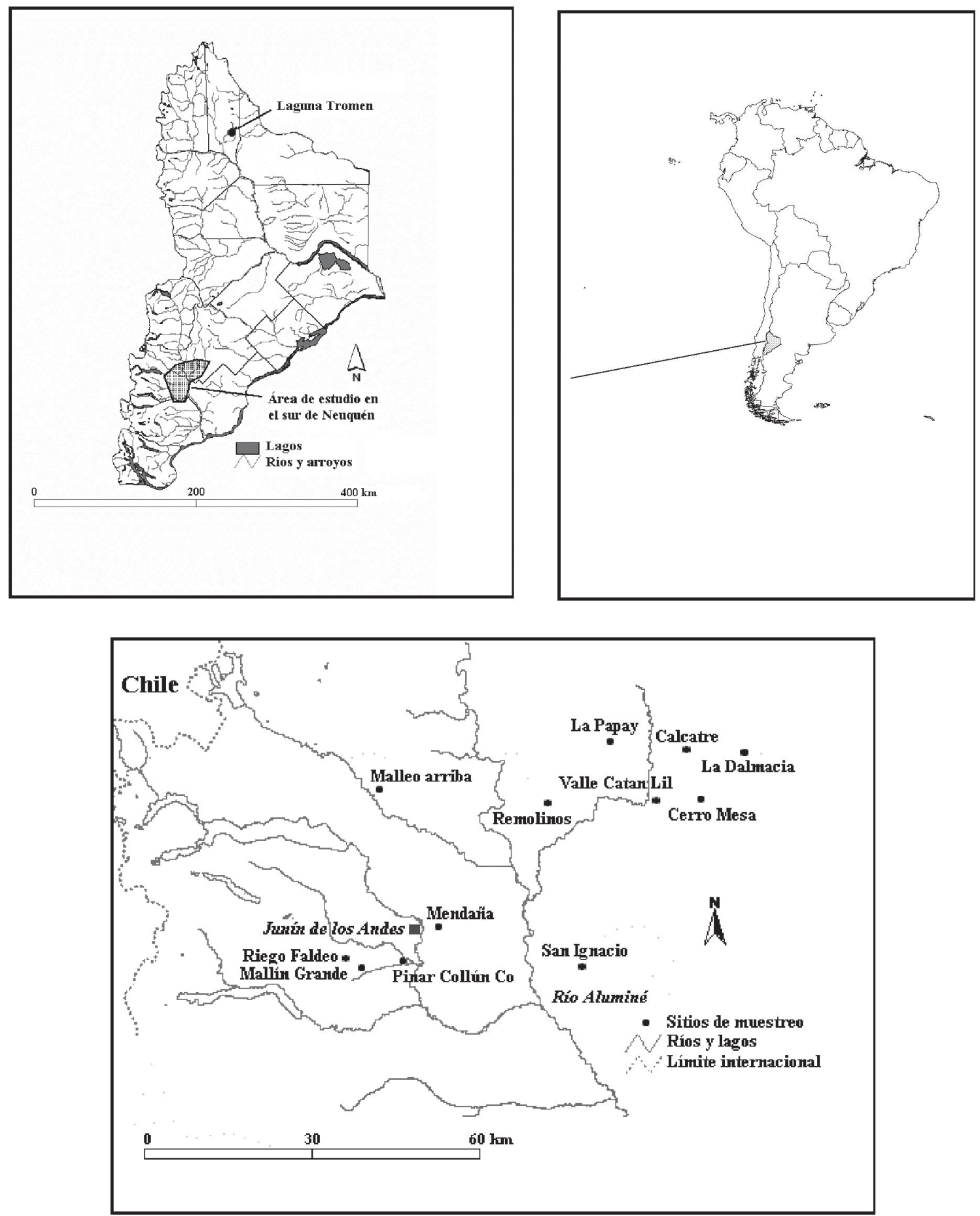

Fig. 1: Arriba: ubicación del área de estudio dentro de la provincia de Neuquén, Argentina, y en Sudamérica. Abajo: detalle de los sitios de estudio en el sur de Neuquén.

Above: study area location in Neuquén Province, Argentina, and in Southamerica. Below: detail of the study sites in southern Neuquén. 
Papay, San Ignacio, Los Remolinos, valle de Catan Lil y La Dalmacia. La vegetación dominante es una estepa arbustivo graminosa con Mullinum spinosum (neneo) y Senecio spp. como arbustos más comunes y diferentes especies de Stipa spp. y Poa spp. como gramíneas predominantes. En esta zona se cría ganado ovino y vacuno. Calcatre y Mesa son sitios con suaves lomadas y mesetas atravesadas por cañadones que bajan al río Catan Lil. La Papay, San Ignacio y La Dalmacia se ubican sobre amplias mesetas a 900-1.200 m de altitud, de las cuales se desprenden cañadones hacia valles circundantes. Los Remolinos también se localiza sobre una meseta elevada pero tiene mayor proporción de "mallines" o zonas húmedas con densa vegetación herbácea de Cortadeira araucana, Juncus spp. y Carex spp. Valle de Catan Lil es el más bajo y abrigado de los sitios orientales, con extensos mallines con alta productividad primaria.

Los sitios más occidentales son Malleo, Riego Faldeo, Mallín Grande, Pinar Collún Co y Mendaña y están ubicados en el ecotono entre la estepa y el bosque subantártico, con predominio de ganadería vacuna. Malleo se sitúa en el valle del río del mismo nombre, es el sitio con precipitaciones más abundantes y su vegetación de ecotono está caracterizada por la presencia de araucarias (Araucaria araucana), habitualmente usadas como perchas por los rapaces. Riego Faldeo, Mallín Grande y Pinar Collún Co son sitios de lomadas suaves con extensos pastizales y mallines y manchones de arbustos y árboles como michay (Berberis spp.), chacay (Chacaya trinervis), maitén (Maytenus boaria) y ñire (Nothofagus antarctica). Mendaña es el sitio más seco entre los occidentales, con $700 \mathrm{~mm}$ de precipitación anual y una menor densidad de árboles y arbustos nativos. Por último, el sitio Tromen se encuentra en el norte de Neuquén a unos 1.500 $\mathrm{m}$ de altitud en las laderas del volcán del mismo nombre, con predominio de vegetación herbácea y ganadería ovina y caprina.

\section{Análisis de las dietas}

Determinamos los hábitos alimenticios de los rapaces a través del análisis de egagrópilas. La recolección de muestras se realizó de manera oportunista en nidos o perchas entre septiembre de 1993 y marzo de 2002, en 13 sitios (véase Área de estudio); 321 egagrópilas (516 ítemes) correspondieron al águila, 115 (390 ítemes) al búho y 78 (193 ítemes) al aguilucho. Dado que las muestras se disgregan con el tiempo, principalmente durante los meses húmedos (otoño e invierno), consideramos que la edad de las egagrópilas no era mayor a seis meses (Marti 1987) y solo incluimos las muestras bien conservadas en el análisis. Una vez colectadas, las muestras se secaron en una estufa, y se disectaron utilizando las técnicas estándares (Marti 1987). Como especímenes de referencia usamos muestras colectadas en el área de estudio (Centro de Ecología Aplicada del Neuquén, CEAN) y colecciones de museo (Museo Argentino de Ciencias Naturales Bernardino Rivadavia, de Buenos Aires). Identificamos a los pequeños mamíferos utilizando claves taxonómicas de pelos (Chehébar \& Martín 1989) y huesos (Pearson 1995). Calculamos el mínimo número de individuos a partir de los pares óseos encontrados en las egagrópilas (mandíbulas y pelvis). Para la cuantificación de artrópodos contamos el número de cabezas encontradas en la muestra, mientras que para aves y reptiles consideramos un solo individuo en la muestra en caso de encontrar plumas o escamas durante el análisis. La nomenclatura sigue a Pearson (1995) para mamíferos y a Dickinson (2003) para aves.

Con el objeto de describir la dieta de los tres rapaces calculamos un índice de diversidad dietario, utilizando la fórmula de Levins (1968):

$$
\mathrm{B}_{\text {sta }}=\left(\mathrm{B}_{\mathrm{obs}}-\mathrm{B}_{\text {mín }}\right) /\left(\mathrm{B}_{\max }-\mathrm{B}_{\min }\right),
$$

donde $\mathrm{B}_{\mathrm{obs}}=1 / \Sigma \mathrm{p}_{\mathrm{i}}{ }^{2}, \mathrm{p}_{\mathrm{i}}$ es la proporción del taxón $\mathrm{i}$ en la dieta de un predador, $\mathrm{B}_{\min }$ es el mínimo posible de diversidad (i.e., 1) y $\mathrm{B}_{\max }$ es el máximo posible (i.e., número total de presas diferentes consumidas por el depredador). Este índice estandarizado permite realizar comparaciones entre las especies. Solo para el águila realizamos una regresión lineal entre el valor obtenido del índice de diversidad dietario y la densidad de liebres en el sitio (véase sección Análisis de datos).

\section{Análisis de datos}

Analizamos la respuesta funcional mediante el estudio de la relación entre el porcentaje de 
ocurrencia de liebres en la dieta de cada rapaz, como un estimador de la tasa de ataque del depredador (Lindén \& Wikman 1983), y la disponibilidad de liebres en el sitio. Esta última fue estimada a partir de la densidad de liebres obtenida de un estudio simultáneo sobre la dinámica poblacional de la liebre en la región (Novaro \& Funes, datos no publicados) en el cual se estimaron densidades utilizando los métodos de conteo de heces (Novaro et al. 1992) y transectas lineales (Novaro et al. 2000). En los casos en que no contamos con estimaciones del mismo sitio, extrapolamos densidades de sitios cercanos con hábitat similares. La densidad de conejo europeo (Oryctolagus cuniculus) en el área de estudio fue mucho menor que la de liebre (Novaro y Funes, observación personal), por lo que se utilizó como disponibilidad solo a la densidad de la última, asumiendo que todos los ítemes presa en la dieta correspondieron a liebre. Para detectar la respuesta funcional hicimos un análisis de regresión lineal simple (Sokal \& Rohlf 1995). Para determinar el tipo de respuesta funcional evaluamos el ajuste de los tres modelos teóricos: lineal, convexo y sigmoideo, a la proporción de liebres consumida por águilas para cada densidad de liebres utilizando el programa STATISTICA (StatSoft, Inc. 2001). No realizamos este ajuste para las otras dos especies de rapaces debido al escaso número de sitios muestreados.

Estimamos la influencia de la liebre en la dieta del águila comparando la composición de la dieta (utilizando frecuencia de los ítemes) entre grupos de sitios con diferente disponibilidad de liebre. Para esto hicimos una prueba de independencia con el estadístico $\mathrm{G}$, a posteriori del análisis de respuesta funcional antes mencionado. Realizamos un análisis similar para el búho y el aguilucho, comparando sus dietas entre sitios, utilizando un criterio de comparación geográfico. Para el búho comparamos la distribución de frecuencias de los ítemes de los dos sitios al sur con el sitio del norte (Tromen), el cual está expuesto a condiciones climáticas más rigurosas y por lo tanto es menos productivo. Para el aguilucho comparamos la dieta en los dos sitios del oeste con la de los dos sitios del este, pretendiendo abarcar en esta comparación el efecto del gradiente de precipitación de la región.
Cuando las diferencias entre los sitios resultaron significativas, evaluamos la contribución a la variación de los ítemes principales con una prueba de $\chi^{2}$ para muestras independientes con partición de grados de libertad y manteniendo una frecuencia mínima de cinco observaciones por celda (Siegel \& Castellan 1995).

Para el águila también realizamos una regresión a posteriori entre el índice de diversidad dietario y la densidad de liebres en cada sitio (Sokal \& Rohlf 1995). No realizamos este análisis con las otras dos especies de rapaces, porque consideramos que el tamaño de la muestra era demasiado pequeño, tomando en cuenta que la liebre no fue la presa principal para ninguna de las dos aves. Los niveles de significancia utilizados siempre fueron iguales al $5 \%$.

\section{RESULTADOS}

\section{Dieta de los rapaces}

En los tres casos estudiados, los mamíferos fueron las presas más frecuentes en las dietas. Los mamíferos nativos constituyeron la mayor parte de la dieta del búho y del aguilucho, siendo los ratones sigmodontinos la presa más importante para cada una de ellas (72 y $42 \%$, respectivamente). La principal presa del águila fue la liebre europea, la que conformó el $35 \%$ del total de ítemes consumidos. La segunda presa vertebrada en orden de importancia por su frecuencia en la dieta, en los tres casos, fue el tucotuco (Ctenomys spp.), roedor de tamaño mediano. Solamente el águila consumió carroña, principalmente ganado menor. El ítem aves estuvo presente en la dieta del águila y del aguilucho, pero no en la dieta del búho (Tablas 1, 2 y 3 ).

El índice de diversidad obtenido siempre fue menor a 0,25 en todos los casos. Considerando todas las presas diferentes consumidas agrupadas al máximo nivel de resolución taxonómica logrado (sin discriminar entre diferentes invertebrados), el aguilucho presentó la dieta más diversa $\left(\mathrm{B}_{\text {sta }}=0,24\right)$ mientras que el búho exhibió la más selectiva $\left(\mathrm{B}_{\text {sta }}=0,18\right)$ y el águila una dieta de diversidad intermedia $\left(\mathrm{B}_{\text {sta }}=0,19\right)$. Un patrón diferente surgió al considerarse solamente las presas vertebradas para el cálculo de este índice, con el búho presentando la dieta más diversa, 


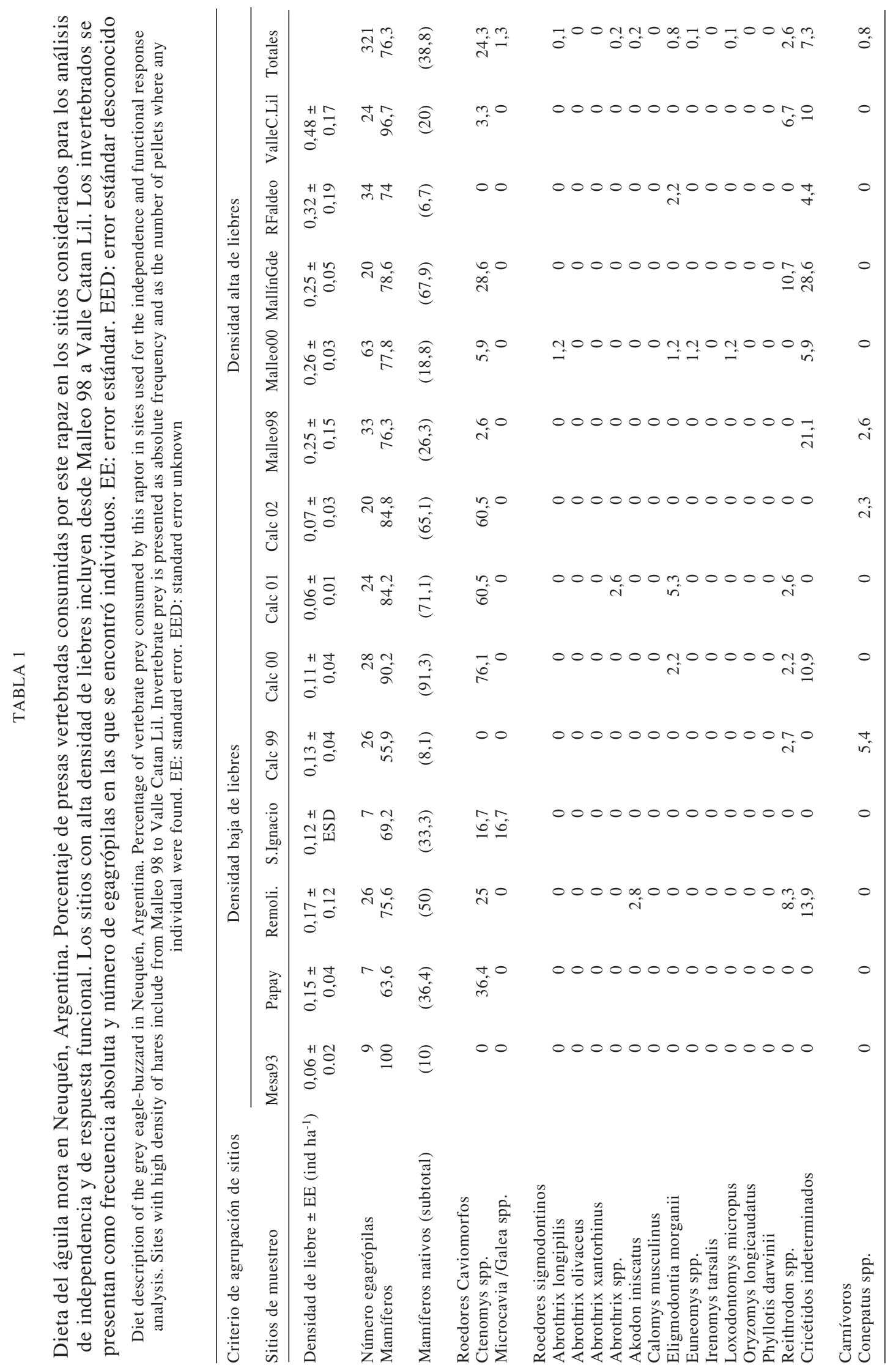




\begin{tabular}{|c|c|c|c|c|c|c|c|c|c|c|c|c|c|c|}
\hline & 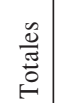 & - & $\overrightarrow{0}$ & $\begin{array}{c}\text { त̂ } \\
\text { fó } \\
\text { fo }\end{array}$ & $n$ & $\vec{m}$ & $\begin{array}{l}+t \\
0 \\
0\end{array}$ & $\begin{array}{l}n \\
0 \\
0\end{array}$ & $+\stackrel{+}{0}=$ & $\stackrel{+}{\infty}$ & 0 & 安 & Fm & $\stackrel{0}{n}$ \\
\hline & 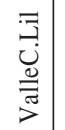 & 0 & 0 & $\underset{E}{E}$ & 0 & ले & $m_{m}^{m}$ & 00 & 000 & $m$ & 0 & iి & mor & $\dot{m}$ \\
\hline 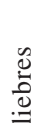 & 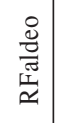 & 0 & 0 & 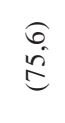 & 0 & $\stackrel{0}{i n}$ & 00 & 00 & $\underset{\forall}{t_{0}} O \underset{\forall}{+}$ & $\dot{\infty}$ & 0 & 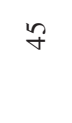 & $m-\sigma$ & in \\
\hline 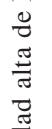 & 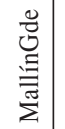 & 0 & 0 & $\stackrel{\overparen{E}}{\ominus}$ & 0 & $\hat{\theta}$ & 00 & 00 & 000 & $\stackrel{\vec{i}}{\vec{i}}$ & 0 & $\stackrel{\infty}{N}$ & 000 & 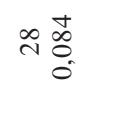 \\
\hline $\begin{array}{l}\overrightarrow{\bar{D}} \\
\overline{0} \\
0\end{array}$ & 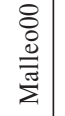 & $\stackrel{\sim}{=}$ & $\stackrel{-}{=}$ & $\begin{array}{l}\tilde{n} \\
\text { है }\end{array}$ & $\stackrel{n}{m}$ & $\begin{array}{l}0 \\
\text { in }\end{array}$ & i & NO & $00=$ & $\stackrel{+}{\sigma}$ & 0 & $\approx$ & $n \mathrm{Nm}$ & \& \\
\hline & $\begin{array}{l}\infty \\
\stackrel{0}{0} \\
\stackrel{\varrho}{=}\end{array}$ & 0 & 0 & कิ & 0 & in & 00 & $0 \underset{i}{0}$ & $0 \underset{i}{0} 0$ & $\underset{\infty}{+}$ & 0 & $\underset{m}{\infty}$ & 000 & $\stackrel{\infty}{m}$ \\
\hline & $\begin{array}{l}\tilde{\delta} \\
u \\
\tilde{J} \\
\tilde{U}\end{array}$ & $\vec{i}$ & 0 & $\begin{array}{l}a \\
a \\
d\end{array}$ & $\stackrel{m}{i}$ & $\hat{\text { हे }}$ & $m_{i} 0$ & O & noo & 0 & 0 & $\stackrel{m}{f}$ & NMo & $\stackrel{+}{q}$ \\
\hline & $\begin{array}{l}\overrightarrow{0} \\
0 \\
0\end{array}$ & 0 & 0 & $\widehat{\hat{n}}$ & 0 & 2 & mo & 00 & $\begin{array}{ll}N & 0 \\
& i\end{array}$ & 0 & 0 & $\stackrel{\infty}{m}$ & 000 & $\stackrel{\infty}{m}$ \\
\hline & $\begin{array}{l}8 \\
0 \\
0\end{array}$ & 0 & 0 & $\underset{\infty}{\infty}$ & 0 & $\stackrel{\circ}{\infty}$ & 00 & 00 & 000 & 0 & 0 & fo & ton & $\vec{n}$ \\
\hline s & gे & 0 & 0 & 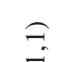 & 0 & $\stackrel{\infty}{\infty}$ & $\stackrel{m}{\infty}^{\circ}$ & $0 \underset{n}{\operatorname{rn}}$ & 000 & $\stackrel{+}{i}$ & 0 & $\hat{n}$ & $\pm \overbrace{N} N$ & in \\
\hline 苞 & चี & & & $\infty$ & & & & & & & & & & \\
\hline 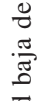 & 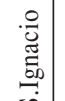 & 0 & 0 & $\stackrel{E}{E}$ & 0 & $\hat{F}$ & 00 & 00 & 000 & $\ddot{\sim}$ & 0 & $\simeq$ & --0 & $=\frac{\hat{a}}{0}$ \\
\hline 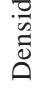 & $\begin{array}{l}: \dot{\overline{0}} \\
\stackrel{0}{0} \\
\simeq\end{array}$ & 0 & 0 & $\begin{array}{l}\text { : } \\
\text { हृ }\end{array}$ & 0 & $a$ & $\begin{array}{l}m \infty \\
\infty \\
\infty\end{array}$ & $\begin{array}{l}\infty \\
i_{i}\end{array}$ & $\underset{i}{\infty} 0$ & $\hat{m}_{\infty}$ & 0 & $\stackrel{n}{n}$ & $\operatorname{tin} 0$ & 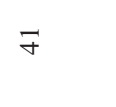 \\
\hline & 总 & 0 & 0 & 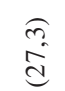 & 0 & $\stackrel{n}{\hat{N}}$ & 00 & 00 & $\begin{array}{l}\text { moo } \\
\stackrel{N}{1}\end{array}$ & $\bar{a}$ & 0 & $=$ & 000 & $=$ \\
\hline & 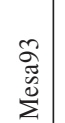 & 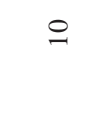 & 0 & 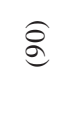 & 0 & లి & 80 & 00 & 000 & 0 & 0 & 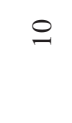 & $n-6$ & $=$ \\
\hline 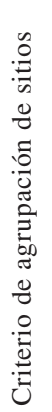 & 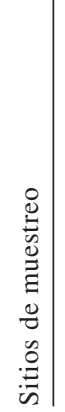 & 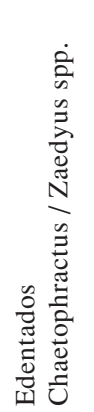 & 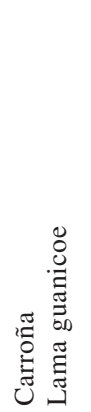 & 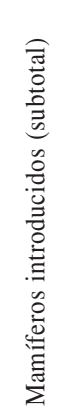 & 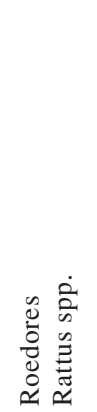 & 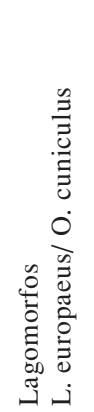 & 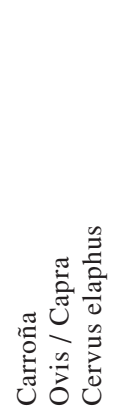 & 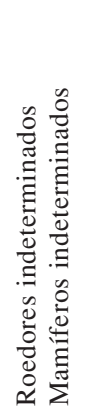 & 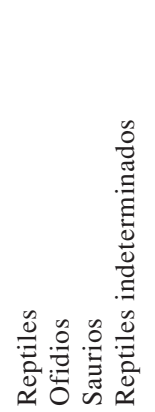 & $\stackrel{\tilde{s}}{\grave{4}}$ & 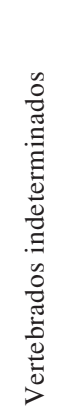 & 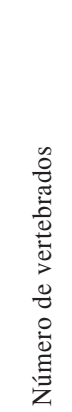 & 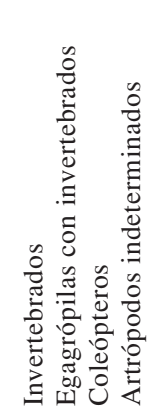 & 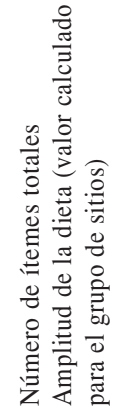 \\
\hline
\end{tabular}


TABLA 2

Dieta del aguilucho común (Buteo polyosoma) en Neuquén, Argentina. Porcentaje de presas vertebradas consumidas por este rapaz en los sitios considerados para los análisis de independencia y de respuesta funcional. Los invertebrados se presentan como frecuencia absoluta y número de egagró-

pilas en las que se encontró individuos. EE: error estándar. EED: error estándar desconocido

Diet description of the red-backed hawk in Neuquén, Argentina. Percentage of vertebrate prey consumed by this raptor in sites used for the independence and functional response analysis. Invertebrate prey is presented as absolute frequency and as the number of pellets where any individual were found. EE: standard error. EED: standard error unknown

\begin{tabular}{|c|c|c|c|c|c|}
\hline \multirow{2}{*}{$\begin{array}{l}\text { Criterio de agrupación de sitios } \\
\text { Sitios de muestreo }\end{array}$} & \multicolumn{2}{|c|}{ Oeste } & \multicolumn{2}{|c|}{ Este } & \multirow[b]{2}{*}{ Totales } \\
\hline & Valle C. Lil 3 & Mendaña & Dalmacia & S. Ignacio 2 & \\
\hline Densidad de liebre $\pm E E\left(\right.$ ind ha $\left.^{-1}\right)$ & $0,8 \pm 0,14$ & $0,25 \pm 0,17$ & $0,29 \pm 0,06$ & $0,12 \pm \mathrm{ESD}$ & \\
\hline Número egagrópilas & 24 & 21 & 13 & 20 & 78 \\
\hline Mamíferos & 90,9 & 72,7 & 84,6 & 81,3 & 82,4 \\
\hline Mamíferos nativos (subtotal) & $(81,8)$ & $(68,2)$ & $(84,6)$ & $(65,6)$ & $(75,1)$ \\
\hline \multicolumn{6}{|l|}{ Roedores caviomorfos } \\
\hline Ctenomys spp. & 51,5 & 15,9 & 46,2 & 18,8 & 33,1 \\
\hline Microcavia /Galea spp. & 0 & 0 & 0 & 0 & 0 \\
\hline \multicolumn{6}{|l|}{ Roedores sigmodontinos } \\
\hline Abrothrix longipilis & 0 & 0 & 0 & 0 & 0 \\
\hline Abrothrix olivaceus & 0 & 0 & 0 & 0 & 0 \\
\hline Abrothrix xantorhinus & 0 & 0 & 0 & 0 & 0 \\
\hline Abrothrix spp. & 6,1 & 0 & 0 & 6,3 & 3,1 \\
\hline Akodon iniscatus & 0 & 0 & 0 & 0 & 0 \\
\hline Calomys musculinus & 3 & 0 & 0 & 0 & 0,8 \\
\hline Eligmodontia morganii & 3 & 6,8 & 15,4 & 0 & 6,3 \\
\hline Euneomys spp. & 0 & 0 & 0 & 0 & 0 \\
\hline Irenomys tarsalis & 0 & 0 & 0 & 0 & 0 \\
\hline Loxodontomys micropus & 0 & 0 & 0 & 0 & 0 \\
\hline Oryzomys longicaudatus & 0 & 0 & 0 & 0 & 0 \\
\hline Phyllotis darwinii & 3 & 0 & 0 & 0 & 0,8 \\
\hline Reithrodon spp. & 0 & 4,5 & 15,4 & 3,1 & 5,8 \\
\hline Cricétidos indeterminados & 15,2 & 40,9 & 7,7 & 37,5 & 25,3 \\
\hline \multicolumn{6}{|l|}{ Carnívoros } \\
\hline Conepatus spp. & 0 & 0 & 0 & 0 & 0 \\
\hline \multicolumn{6}{|l|}{ Edentados } \\
\hline Chaetophractus / Zaedyus spp. & 0 & 0 & 0 & 0 & 0 \\
\hline \multicolumn{6}{|l|}{ Carroña } \\
\hline Lama guanicoe & 0 & 0 & 0 & 0 & 0 \\
\hline Mamíferos introducidos (subtotal) & $(9,1)$ & $(2,3)$ & (0) & $(9,4)$ & $(5,2)$ \\
\hline \multicolumn{6}{|l|}{ Roedores } \\
\hline Rattus spp. & 0 & 0 & 0 & 6,3 & 1,6 \\
\hline \multicolumn{6}{|l|}{ Lagomorfos } \\
\hline Lepus europaeus/Oryctolagus cuniculus & 9,1 & 2,3 & 0 & 3,1 & 3,6 \\
\hline \multicolumn{6}{|l|}{ Carroña } \\
\hline Ovis / Capra & 0 & 0 & 0 & 0 & 0 \\
\hline Cervus elaphus & 0 & 0 & 0 & 0 & 0 \\
\hline Roedores indeterminados & 0 & 2,3 & 0 & 6,3 & 2,1 \\
\hline Mamíferos indeterminados & 0 & 0 & 0 & 0 & 0 \\
\hline
\end{tabular}


TABLA 2 (continuación)

\begin{tabular}{|c|c|c|c|c|c|}
\hline \multirow{2}{*}{$\begin{array}{l}\text { Criterio de agrupación de sitios } \\
\text { Sitios de muestreo }\end{array}$} & \multicolumn{2}{|c|}{ Oeste } & \multicolumn{2}{|c|}{ Este } & \multirow[b]{2}{*}{ Totales } \\
\hline & Valle C. Lil 3 & Mendaña & Dalmacia & S. Ignacio 2 & \\
\hline \multicolumn{6}{|l|}{ Reptiles } \\
\hline Ofidios & 0 & 2,3 & 11,5 & 0 & 3,5 \\
\hline Saurios & 3 & 4,5 & 3,8 & 3,1 & 3,6 \\
\hline Reptiles indeterminados & 0 & 18,2 & 0 & 15,6 & 8,5 \\
\hline Aves & 6,1 & 2,3 & 0 & 0 & 2,1 \\
\hline Vertebrados indeterminados & 0 & 0 & 0 & 0 & 0 \\
\hline Número de vertebrados & 33 & 44 & 26 & 32 & 135 \\
\hline \multicolumn{6}{|l|}{ Invertebrados } \\
\hline Egagrópilas con invertebrados & 7 & 7 & 8 & 5 & 27 \\
\hline Coleópteros & 2 & 11 & 3 & 5 & 21 \\
\hline Artrópodos indeterminados & 7 & 1 & 28 & 1 & 37 \\
\hline Número de ítemes totales & 42 & 56 & 57 & 38 & 193 \\
\hline $\begin{array}{l}\text { Amplitud de la dieta } \\
\text { (valor calculado para el grupo } \\
\text { de sitios) }\end{array}$ & \multicolumn{2}{|r|}{0,276} & & 0,193 & \\
\hline
\end{tabular}

TABLA 3

Dieta del búho magallánico (Bubo magellanicus) en Neuquén, Argentina. Porcentaje de presas vertebradas consumidas por este rapaz en los sitios considerados para los análisis de independencia y de respuesta funcional. Los invertebrados se presentan como frecuencia absoluta y número de egagrópilas en las que se encontró individuos. EE: error estándar

Diet description of the Magellanic horned owl in Neuquén, Argentina. Percentage of vertebrate prey consumed by this raptor in sites used for the independence and functional response analysis. Invertebrate prey is presented as absolute frequency and as the number of pellets where any individual were found. EE: standard error

\begin{tabular}{|c|c|c|c|c|}
\hline \multirow{2}{*}{$\begin{array}{l}\text { Criterio de agrupación de sitios } \\
\text { Sitios de muestreo }\end{array}$} & \multicolumn{2}{|c|}{ Sur } & \multicolumn{2}{|c|}{ Norte } \\
\hline & Pinar Collún Co & Valle C. Lil 2 & Tromen & Totales \\
\hline Densidad de liebre \pm EE $\left(\right.$ ind $\left.h^{-1}\right)$ & $0,14 \pm 0,08$ & $0,58 \pm 0,11$ & $0,22 \pm 0,11$ & \\
\hline Número egagrópilas & 26 & 15 & 74 & 115 \\
\hline Mamíferos & 100 & 97,4 & 98,1 & 98,5 \\
\hline Mamíferos nativos (subtotal) & $(96,4)$ & $(92,3)$ & $(87,3)$ & $(92)$ \\
\hline \multicolumn{5}{|l|}{ Roedores caviomorfos } \\
\hline Ctenomys spp. & 12,7 & 25,6 & 19,7 & 19,4 \\
\hline Microcavia /Galea spp. & 0 & 0 & 0,6 & 0,2 \\
\hline \multicolumn{5}{|l|}{ Roedores sigmodontinos } \\
\hline Abrothrix longipilis & 1,8 & 0 & 4,5 & 2,1 \\
\hline Abrothrix olivaceus & 5,5 & 0 & 1,9 & 2,5 \\
\hline Abrothrix xantorhinus & 0 & 0 & 0,6 & 0,2 \\
\hline Abrothrix spp. & 0 & 0 & 2,5 & 0,8 \\
\hline Akodon iniscatus & 1,8 & 0 & 0,6 & 0,8 \\
\hline Calomys musculinus & 3,6 & 7,7 & 0,6 & 4 \\
\hline Eligmodontia morganii & 12,7 & 7,7 & 18,5 & 13 \\
\hline Euneomys spp. & 0 & 0 & 5,1 & 1,7 \\
\hline Irenomys tarsalis & 1,8 & 0 & 0 & 0,6 \\
\hline Loxodontomys micropus & 9,1 & 0 & 0,6 & 3,2 \\
\hline Oryzomys longicaudatus & 1,8 & 10,3 & 0 & 4 \\
\hline Phyllotis darwinii & 1,8 & 12,8 & 0 & 4,9 \\
\hline Reithrodon spp. & 16,4 & 12,8 & 7 & 12,1 \\
\hline Cricétidos indeterminados & 27,3 & 15,4 & 24,8 & 22,5 \\
\hline \multicolumn{5}{|l|}{ Carnívoros } \\
\hline Conepatus spp. & 0 & 0 & 0 & 0 \\
\hline
\end{tabular}


TABLA 3 (continuación)

\begin{tabular}{|c|c|c|c|c|}
\hline \multirow{2}{*}{$\begin{array}{l}\text { Criterio de agrupación de sitios } \\
\text { Sitios de muestreo }\end{array}$} & \multicolumn{2}{|c|}{ Sur } & \multicolumn{2}{|c|}{ Norte } \\
\hline & Pinar Collún Co & Valle C. Lil 2 & Tromen & Totales \\
\hline \multicolumn{5}{|l|}{ Edentados } \\
\hline Chaetophractus / Zaedyus spp. & 0 & 0 & 0 & 0 \\
\hline \multicolumn{5}{|l|}{ Carroña } \\
\hline Lama guanicoe & 0 & 0 & 0 & 0 \\
\hline Mamíferos introducidos (subtotal) & $(0)$ & $(2,6)$ & (7) & $(3,2)$ \\
\hline \multicolumn{5}{|l|}{ Roedores } \\
\hline Rattus spp. & 0 & 0 & 2,5 & 0,8 \\
\hline \multicolumn{5}{|l|}{ Lagomorfos } \\
\hline L. europaeus/O. cuniculus & 0 & 2,6 & 4,5 & 2,3 \\
\hline \multicolumn{5}{|l|}{ Carroña } \\
\hline Ovis / Capra & 0 & 0 & 0 & 0 \\
\hline Cervus elaphus & 0 & 0 & 0 & 0 \\
\hline Roedores indeterminados & 3,6 & 2,6 & 3,8 & 3,3 \\
\hline Mamíferos indeterminados & 0 & 0 & 0 & 0 \\
\hline \multicolumn{5}{|l|}{ Reptiles } \\
\hline Ofidios & 0 & 0 & 0,6 & 0,2 \\
\hline Saurios & 0 & 2,6 & 0,6 & 1,1 \\
\hline Reptiles indeterminados & 0 & 0 & 0 & 0 \\
\hline Aves & 0 & 0 & 0 & 0 \\
\hline Vertebrados indeterminados & 0 & 0 & 0,6 & 0,2 \\
\hline Número de vertebrados & 55 & 39 & 157 & 251 \\
\hline \multicolumn{5}{|l|}{ Invertebrados } \\
\hline Egagrópilas con invertebrados & 0 & 3 & 135 & 130 \\
\hline Coleópteros & 0 & 3 & 90 & 93 \\
\hline Artrópodos indeterminados & 0 & 1 & 45 & 46 \\
\hline Número de ítemes totales & 55 & 43 & 292 & 390 \\
\hline $\begin{array}{l}\text { Amplitud de la dieta (valor calculado } \\
\text { para el grupo de sitios) }\end{array}$ & \multicolumn{2}{|c|}{0,363} & \multicolumn{2}{|c|}{0,104} \\
\hline
\end{tabular}

seguido por el aguilucho y luego por el águila $\left(\mathrm{B}_{\text {sta }}=0,33,0,24\right.$ y 0,15 , respectivamente $)$.

El índice sugirió que el águila expande su dieta en los sitios en donde la densidad de liebre es baja, y el aguilucho hace lo mismo en los sitios al oeste. Sin embargo, la regresión lineal entre el índice y la densidad de liebres en cada sitio para el águila resultó ser no significativa, tanto al considerar todos los ítemes de su dieta como al incluir solo las presas vertebradas $(\mathrm{P}=$ $0,53, \mathrm{R}^{2}=0,04$, mismo valor en ambos casos).

\section{Respuestas funcionales}

El águila es el único de los tres rapaces que presentó un incremento significativo en el consumo de liebres con relación a densidades crecientes de liebre $\left(n=13, R^{2}=0,50, P<0,01\right)$.
Las respuestas funcionales del aguilucho $(\mathrm{n}=4$, $\left.\mathrm{R}^{2}=0,8036, \mathrm{P}=0,10\right)$, así como la del búho $(\mathrm{n}=$ $\left.3, \mathrm{R}^{2}=0,07, \mathrm{P}=0,83\right)$ no fueron detectadas en este estudio. El águila presentó una saturación en su respuesta funcional a partir de una densidad aproximada de 0,3 liebres por hectárea, con una frecuencia de 65-75\% de liebre (Fig. 2). El ajuste de los tres modelos no nos permite definir cuál modelo explica mejor los datos ya que, excepto por la asíntota del modelo sigmoideo, ninguno de los parámetros estimados difirió significativamente de cero (Tabla 4).

\section{Cambios de dieta entre sitios}

El águila cambió sus hábitos alimenticios dependiendo del sitio $(\mathrm{G}=129,9, \mathrm{P}<0,0001)$, consumiendo diferencialmente todos los ítemes 

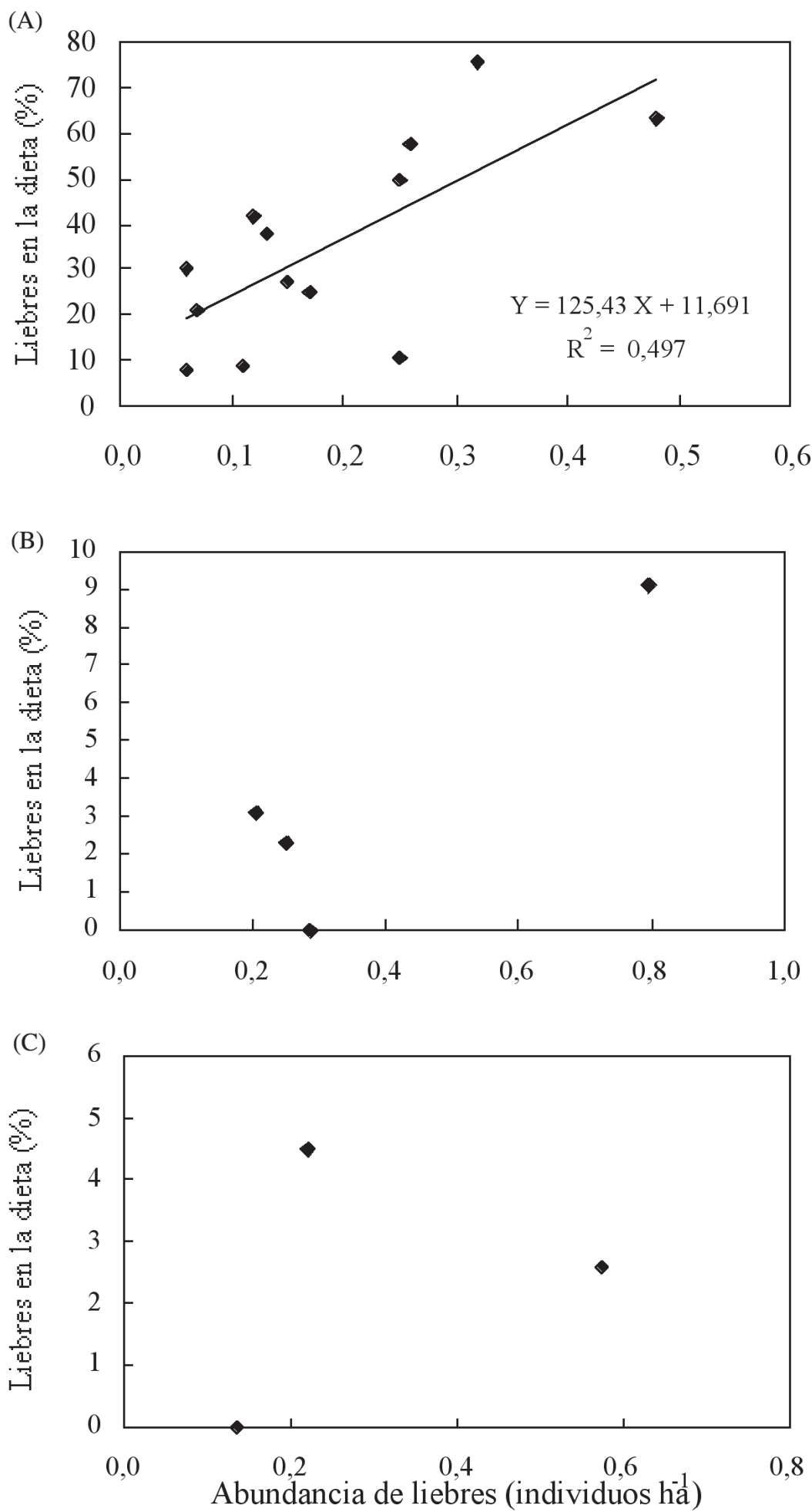

Fig. 2: Respuesta funcional de tres especies de aves rapaces frente a la liebre europea en Neuquén, noroeste de Patagonia, Argentina. (A) Respuesta del águila. La ecuación de la regresión y $\mathrm{R}^{2}$ se muestra dentro del área de gráfico. (B) Respuesta del aguilucho. (C) Respuesta del búho.

Functional response of three raptor species to the European hare in Neuquén, northwestern Patagonia, Argentina. (A) Grey eagle-buzzard response. Regression equation and $\mathrm{R}^{2}$ are shown inside the graphic area. (B) Red-backed hawk response. (C) Magellanic horned-owl response 


\section{TABLA 4}

Parámetros de los distintos modelos ajustados a los datos de respuesta funcional del águila en Neuquén, Argentina. Se presentan en negrita los valores que se esperaban significativos. El valor con asterisco presenta aquel que efectivamente fue significativo

Different models parameters adjusted to the functional response data of the grey eagle-buzzard in Neuquén, Argentina. The values presented in bold are those expected to be significant. The value with the asterisk presents the one that indeed was significant

\begin{tabular}{|c|c|c|c|c|c|c|c|c|c|}
\hline & \multicolumn{3}{|c|}{ Modelo sigmoideo } & \multicolumn{3}{|c|}{ Modelo convexo } & \multicolumn{3}{|c|}{ Modelo lineal (piecewise) } \\
\hline & Asíntota & Pendiente & Intersección & Pendiente & Constante & $\begin{array}{l}\text { Ordenada } \\
\text { al origen }\end{array}$ & $\begin{array}{l}\text { Pendiente } \\
\text { inicial }\end{array}$ & Inflexión & $\begin{array}{l}\text { Pendiente } \\
\text { del plateau }\end{array}$ \\
\hline Valor estimado & 75,62 & 5,25 & $-8,22$ & 136,62 & 0,5 & 13,45 & 104,31 & 100,07 & $-76,6$ \\
\hline Error estándar & 28,89 & 3,57 & 5,26 & 122,84 & 0,68 & 10,6 & 64,22 & 55,74 & 136,65 \\
\hline Estadístico t & 2,62 & 1,47 & $-1,56$ & 1,11 & 0,73 & 1,27 & 1,62 & 1,8 & $-0,56$ \\
\hline Valor de P & $0,03 *$ & 0,17 & 0,15 & 0,29 & 0,48 & 0,24 & 0,14 & 0,11 & 0,59 \\
\hline Grados de libertad & & 10 & & & 11 & & & 9 & \\
\hline Valor de R & & 0,72 & & & 0,7 & & & 0,79 & \\
\hline$\%$ varianza explicada & & 51,54 & & & 49,38 & & & 61,83 & \\
\hline
\end{tabular}

considerados para el análisis $(\mathrm{P}<0,005)$ : tucotuco, liebre, roedores sigmodontinos, aves, carroña (ganado menor) y reptiles. Los artrópodos podrían haber sido incidentalmente ingeridos, por lo cual no fueron incluidos en el análisis, dado que podrían enmascarar los patrones de los demás ítemes. La mayor parte de la varianza fue explicada por el mayor consumo de liebres en sitios donde la misma es más abundante, como se observa en los resultados arrojados por la partición de la tabla de contingencia $\left(X^{2}=57,3\right)$. En los sitios de baja densidad de liebre, el águila consumió principalmente tuco-tucos entre las presas nativas $\left(\mathrm{X}^{2}=38\right)$, y también más carroña $\left(\mathrm{X}^{2}=\right.$ 14,9).

También el aguilucho varió la composición de su dieta entre sitios $(\mathrm{G}=18,3, \mathrm{P}<0,005)$. Consideramos los mismos grupos de ítemes que en el caso del águila, excepto por la carroña que no fue considerada por estar ausente en la dieta de este rapaz. La proporción de roedores nativos explicó la mayor parte de la variación entre sitios. El análisis de partición de grados de libertad demostró que el aguilucho consume mayor cantidad de tuco-tucos en el este, y menor cantidad de roedores sigmodontinos $\left(\mathrm{X}^{2}\right.$ $=20,24, \mathrm{P}<0,005)$. Las proporciones del resto de los ítems no explicaron una cantidad significativa de variación de la dieta entre sitios $\left(\mathrm{X}^{2}=0,003, \mathrm{P}>0,05\right)$.

En el caso del búho, no hubo diferencias significativas entre la dieta en los sitios al norte $y$ al sur $(G=3,27, P>0,05)$. Los ítemes que consideramos para este análisis fueron tucotucos, roedores sigmodontinos, liebre y reptiles. La frecuencia de ocurrencia del resto de los ítemes no fue suficiente como para incluirlos en el análisis.

\section{DISCUSIÓN}

\section{Dieta de las rapaces}

Actualmente la principal presa del águila en el área de estudio es la liebre europea. Estudios previos en la región muestran este mismo comportamiento, reportando siempre un lagomorfo como presa principal de este rapaz (Pavez et al. 1992, Hiraldo et al. 1995). El cambio de consumo de presas nativas a introducidas no es exclusivo de las aves rapaces en la región (Franklin et al. 1999, Novaro et al. 2000). Novaro et al. (2000) encontraron un patrón similar entre los carnívoros terrestres de la zona, con lo que es evidente el impacto de las especies introducidas en las comunidades del noroeste de Patagonia.

El águila es el rapaz que presenta el mayor número de ítemes presa diferentes y a la vez el menor índice de diversidad de presas vertebradas, lo cual sugiere que podría tener mayor capacidad para cazar diferentes presas, pero que concentra su dieta en ciertos ítemes, aparentemente los más redituables desde el punto de vista energético. Tanto el aguilucho como el búho son capaces de consumir liebres 
europeas, pero en ambos casos sus presas principales fueron pequeños roedores nativos. Pese a que la liebre es una presa más redituable en cuanto a su peso, la mayor proporción de tuco-tuco en la dieta del aguilucho y del búho, posiblemente se deba a la dificultad de estos rapaces para cazar liebres debido a su pequeño tamaño corporal. Si los roedores pequeños son más abundantes que los roedores de mediano tamaño como los tuco-tucos, esto explicaría el mayor consumo de los primeros por ambos rapaces según, la teoría de forrajeo óptimo (Schoener 1971).

La dieta del aguilucho muestra que este es el depredador más generalista de los tres estudiados. El aguilucho posee una dieta menos diversa en los sitios del este del área de estudio, donde posiblemente el tuco-tuco es más abundante. El águila presenta la misma tendencia en su comportamiento alimentario, con una dieta menos diversa en los sitios donde la liebre es más abundante, aunque el resultado no significativo de la regresión lineal sugiere que este patrón puede ser no lineal.

\section{Respuestas funcionales}

Pavez et al. (1992) e Hiraldo et al. (1995) sugirieron la existencia de una respuesta funcional en el águila con respecto al conejo y la liebre europea, respectivamente, cuando detectaron variaciones temporales en la frecuencia de dichos lagomorfos en la dieta del águila. Nuestro estudio es el primero en cuantificar dicha respuesta funcional. No detectamos respuestas funcionales respecto a la liebre europea en el comportamiento alimentario del aguilucho y del búho. Aunque nuestro número de muestras en ambos casos es demasiado pequeño para realizar conclusiones definitivas, los resultados sugieren que la disponibilidad de libres puede no tener un efecto importante en la dieta de estos rapaces debido a su talla. Detectamos como principal presa del búho a los roedores sigmodontinos al igual que Trejo \& Grigera (1998). Sin embargo, no podemos descartar que este resultado refleje simplemente el consumo por un depredador oportunista de las presas más abundantes, pues no poseemos datos de disponibilidad de estos roedores en todos los sitios de estudio.

Numerosos estudios previos en aves rapaces encontraron que la curva de respuesta funcional llega a un techo o plateau, indicando la saturación del depredador. Sin embargo, diferentes autores han ajustado diversos modelos a la respuesta funcional de águilas, correspondiendo en algunos casos al tipo convexo, típico de depredadores especialistas (Restani et al. 2000, Salamolard et al. 2000). No obstante, una respuesta funcional sigmoidea puede verse enmascarada cuando la densidad de la presa principal cambia más abruptamente que la de la presa alternativa, obteniéndose una respuesta del tipo convexo, con lo que las consecuencias de la depredación en la población de presas no deberían ser inferidas únicamente a partir de la curva de respuesta ante la presa principal (Murdoch 1973). Aunque encontramos una respuesta funcional significativa para el águila, no podemos decidir sobre la base de nuestros resultados, qué modelo de respuesta explica mejor el comportamiento del águila, posiblemente debido a un insuficiente número de sitios de muestreo. Jaksic et al. (1992) no encontraron una respuesta funcional significativa en aves depredadoras estudiadas en Chile, pero sí una fuerte respuesta numérica ante las fluctuaciones de pequeños mamíferos. En nuestro caso no evaluamos la respuesta numérica de los rapaces, pero cabe mencionar que el águila parece ser fuertemente territorial (SánchezAldao et al. 2001), siendo esta una característica que limitaría una respuesta numérica por migración como la reportada por Jaksic et al. (1992). No obstante, es posible que este carácter territorial de las águilas se restrinja a las estaciones reproductivas. $\mathrm{Si}$ el tamaño poblacional de este rapaz se mantiene relativamente constante a través del año, se reforzaría el efecto de depredación del águila sobre la población de liebres. Es posible, por otro lado, que la depredación por parte del águila contribuya a la regulación de la población de liebre europea al sumarse al consumo de liebres que realizan los mamíferos depredadores de la zona, como el zorro culpeo (Pseudalopex culpaeus), el zorro chilla ( $P$. griseus) y el puma (Puma concolor) (Novaro et al. 2000).

\section{Comparación de la dieta entre sitios}

La dieta del águila varía marcadamente en función de la densidad de liebres de cada sitio. 
Esta respuesta implica un comportamiento generalista, por el cual el consumo de cada presa depende no solo de su disponibilidad sino también de la disponibilidad de liebres. Este mecanismo suele estar ligado a un tipo de respuesta funcional sigmoidea frente a la presa principal, posibilitando la regulación poblacional de la misma por depredación (Murdoch 1973, Schmitz 1995).

El aguilucho mostró también un comportamiento alimentario generalista, con diferencias en su dieta entre los sitios orientales y occidentales del área de estudio. Esta variación es explicada por el consumo diferencial de roedores nativos, siendo en el oeste su principal presa los sigmodontinos, pero presentando en el este un mayor consumo de tuco-tucos. En la zona oriental del área de estudio, los suelos habitualmente son más arenosos y menos consolidados y por ende más favorables para especies fosoriales como los tuco-tucos, cuyas cuevas son observadas con mayor frecuencia (Funes y Novaro, observación personal). Por lo tanto, el aguilucho podría estar depredando más tuco- tucos donde estos son más abundantes, dado que es una presa más redituable energéticamente que los sigmodontinos, debido a su mayor tamaño. Por otra parte, no se observaron diferencias marcadas en la dieta del búho entre sitios. No podemos concluir sobre la base de este resultado que exista un comportamiento especialista de este depredador porque no poseemos datos de disponibilidad de sus principales presas. Sin embargo, es muy poco probable que los ensambles de presas disponibles para el búho sean los mismos en los sitios comparados dado que son ambientes separados geográficamente y notablemente diferentes.

\section{AGRADECIMIENTOS}

Agradecemos al departamento de Fauna Terrestre del CEAN (Centro de Ecología Aplicada del Neuquén) por brindar materiales de trabajo y el laboratorio para el análisis de las egagrópilas, y en particular a Guillermo Sánchez Aldao, Oscar Pailacura y Obdulio Monsalvo por la recolección de egagrópilas. Agradecemos a Susan Walker, quien confeccionó los mapas del área de estudio y asistió con los análisis estadísticos, y a los dos revisores anónimos que brindaron sugerencias para mejorar el manuscrito. También agradecemos el espacio cedido en el Museo Argentino de Ciencias Naturales Bernardino Rivadavia. Las tareas de campo fueron subvencionadas por el CEAN, la Secretaría de Agricultura, Ganadería, Pesca y Alimentación de la Nación y la Wildlife Conservation Society.

\section{LITERATURA CITADA}

CABRERA AL (1976) Regiones fitogeográficas argentinas. Enciclopedia Argentina de agricultura y Jardinería. Segunda Edición, Tomo II, Fase 1 ACME, Buenos Aires, Argentina. 85 pp.

CHEHÉBAR C \& S MARTÍN (1989) Guía para el reconocimiento microscópico de los pelos de los mamíferos de la Patagonia. Doñana, Acta Vertebrata (España) 16: 247-291.

DAVIS SA, H LEIRS, R P PECH, Z ZHANG \& NC STENSETH (2003) On the economic benefit of predicting rodent outbreaks in agricultural systems. Crop Protection 23: 305-314.

DICKINSON E. ed (2003) The Howard and Moore complete checklist of the birds of the world. Princeton University Press, Princeton, New Jersey, USA. $1056 \mathrm{pp}$.

DONÁZAR JA, A TRAVAINI, O CEBALLOS, M DELIBES \& F HIRALDO (1997) Food habits of the great horned owl in northwestern Argentine Patagonia: the role of introduced lagomorphs. Journal of Raptor Research (USA)31: 364-369.

ERLINGE S, G GORANSSON, G HOGSTEDT, G JANSSON, O LIBERG, J LOMAN, IN NILSSON, T VON SCHANTZ \& M SYLVÉN (1984) Can vertebrate predators regulate their prey? American Naturalist 123: 125-133.

FRANKLIN WL, WE JOHNSON, RJ SARNO \& JA IRIARTE (1999) Ecology of the Patagonia puma Felis concolor patagonica in southern Chile. Biological Conservation 90: 33-40.

GRIGERA DE \& EH RAPOPORT (1983) Status and distribution of the European hare in South America. Journal of Mammalogy 64: 163-166.

HIRALDO F, JA DONÁZAR, O CEBALLOS, A TRAVAINI, J BUSTAMANTE \& MC FUNES (1995) Breeding biology of the grey eagle-buzzard population in Patagonia. Wilson Bulletin 107: 675-685.

HOLLING CS (1959) Some characteristics of simple types of predation and parasitism. Canadian Entomologist 91: 385-398.

JAKSIC FM, JE JIMÉNEZ, SA CASTRO \& P FEINSINGER (1992) Numerical and functional response of predators to a long-term decline in mammalian prey at semi-arid Neotropical site. Oecologia 89: 90-101.

JIMÉNEZ JE \& FM JAKSIC (1990) Historia natural de Geranoaetus melanoleucus. Hornero 13: 97-110.

JIMÉNEZ JE \& FM JAKSIC (1991) Behavioural ecology of red-backed hawks in central Chile. Wilson Bulletin 103: 132-137.

KORPIMÄKI E \& K NORRDAHL (1989) Predation of Tengmalm's owls: numerical responses, functional responses and dampening impact on population fluctuations of microtines. Oikos 54:154-164. 
KORPIMÄKI E \& K NORRDAHL (1991) Numerical and functional responses of kestrels, Short-eared owls, and long-eared owls to voles densities. Ecology 72: $814-826$

LEVINS R (1968) Evolution in changing environments. Princeton University Press, Princeton, New Jersey, USA. 120 pp.

LINDÉN H \& M WIKMAN (1983) Goshawk predation on tetraonids: availability of prey and diet of the predator in the breeding season. Journal of Animal Ecology 52: 953-968.

MARTI CD (1987) Raptor food habits studies. National Wildlife Federation, Raptor management techniques manual: 67-80. Washington, District of Columbia, USA.

MURDOCH WW (1973) The functional response of predators. Journal of Applied Ecology 10: 335 342.

NORRDAHL K \& E KORPIMÄKI (2000) Do predators limit the abundance of alternative prey? Experiments with vole-eating avian and mammalian predators. Oikos 91: 528-540.

NOVARO AJ, MC FUNES \& RS WALKER (2000) Ecological extinction of native prey of a carnivore assemblage in Argentine Patagonia. Biological Conservation 92: 25-33

NOVARO AJ, AF CAPURRO, A TRAVAINI, MC FUNES \& JE RABINOVICH (1992) Pellet-count sampling based on spatial distribution: a case study of the European hare in Patagonia. Ecología Austral (Argentina) 2: 11-18.

PAVEZ EF, CA GONZÁLEZ \& JE JIMÉNEZ (1992) Diet shifts of Black-chested Eagles (Geranoaetus melanoleucus) from native prey to European Rabbits in Chile. Journal of Raptor Research (USA) 26: $27-32$

PEARSON OP (1995) Annotated keys for identifying small mammals living in or near Nahuel Huapi National Park or Lanín National Park, southern Argentina. Mastozoología Neotropical (Argentina) 2: 99-148.
RESTANI M, AR HARMATA \& EM MADDEN (2000) Numerical and functional responses of migrant bald eagles exploiting a seasonally concentrated food source. Condor 102: 561-568.

SALAMOLARD M, A BUTET, A LEROUX \& V BRETAGNOLLE (2000) Responses of an avian predator to variations in prey density at a temperate latitude. Ecology 81: 2428-2441.

SÁNCHEZ-ALDAO G, MC FUNES, OB MONSALVO, MB BONGIORNO \& O PAILACURA (2001) El águila mora en el sudeste del Departamento Huiliches, Provincia del Neuquén. Departamento de Fauna Silvestre Terrestre, Centro de Ecología Aplicada del Neuquén, Argentina. 11 pp.

SCHMITZ OJ (1995) Functional responses of optimal consumers and the potential for regulation of resource populations. Wildlife Research 22: 101-113.

SCHOENER TW (1971) Theory of feeding strategies. Annual Review of Ecology and Systematics 11: 396-404.

SIEGEL S \& NJ CASTELLAN (1995) Estadística no paramétrica: aplicada a las ciencias de la conducta. Cuarta edición. Editorial Trillas, México. 437 pp.

SOKAL RR \& FJ ROHLF (1995) Biometry: the principles and practice of statistics in biological research. Third edition. WH Freeman and Company, New York, New York, USA. 850 pp.

SOLOMON ME (1949) The natural control of animal populations. Journal of Animal Ecology 18: 1-35.

STATSOFT, INC (2001) STATISTICA (data analysis software system), version 6. www.statsoft.com.

TREJO A \& D GRIGERA (1998) Food habits of the great horned owl (Bubo virginianus) in a Patagonian steppe in Argentina. Journal of Raptor Research (USA) 32: 306-311.

WOOD BJ \& CHUNG GAIT FEE (2003) A critical review of the development of rat control in Malaysian agriculture since the 1960s. Crop Protection 22: 445-461. 